\title{
Hyper-IgE syndrome: A rare cause of recurrent infections in children
}

\author{
Elena-Lia Spoiala ${ }^{1,2}$, Aniela-Luminita Rugina ${ }^{1,2}$, Aurica Rugina ${ }^{1,2}$ \\ 1"Sf. Maria" Emergency Clinical Hospital for Children, Iasi, Romania \\ 2"Gr.T. Popa" University of Medicine and Pharmacy, lasi, Romania
}

\begin{abstract}
Hyperimmunoglobulinemia $E$ or hyper-IgE syndrome (HIES) is a complex primary immunodeficiency disorder characterized by recurrent skin and pulmonary infections, eczema, eosinophilia, and high serum levels of IgE. The exact prevalence of HIES remains unknown, although this condition is considered to affect less than 1 per million people worldwide. We present a case-report of a 9 year-old girl, symptomatic since early infancy, with distinctive facial features (broad nasal bridge, antimongoloid palpebral fissures), eczema, persistence of primary teeth and recurrent bacterial infections (skin and pulmonary abscesses) due to Staphyloccocus aureus. The diagnosis of HIES was established according to National Institutes of Health scoring system based on both clinical and laboratory findings. Despite antibacterial prophylaxis with trimethoprim-sulfamethoxazole, the patient developed a staphylococcal abscess of right temporal region one year after the last admission in the hospital.
\end{abstract}

Keywords: hyperimmunoglobulinemia E, recurrent infections, children

\section{INTRODUCTION}

HIES is a rare, genetic disorder characterized by chronic eczema, eosinophilia and high serum levels of IgE. This triad is also present in atopic conditions which can lead to difficulties of diagnosis. However, HIES should be considered in patients with recurrent infections with frequent involvement of skin, sinuses and lungs, neonatal onset dermatitis and a variety of connective tissue, vascular and skeletal abnormalities. The laboratory findings include high serum levels of IgE, usually more than $2,000 \mathrm{IU} / \mathrm{ml}$. HIES was first described as Job syndrome in 1966 in two children with severe dermatitis, fair skin, recurrent staphylococcal infections, abnormal inflammatory response, and hyperextensible joints (1). The association with markedly elevated serum IgE levels and eosinophilia was noticed in 1972 when Buckley et. al reported the cases of two boys with severe dermatitis, recurrent cutaneous, pulmonary and articular abscesses, growth failure, and coarse facies (2). Beginning with 2007, remarkable progress has been made in understanding of the genetic basis of the disease. Although most cases of HIES are sporadic ones, autosomal dominant and recessive inheritance patterns have also been reported. The autosomal dominant HIES, also known as
Job syndrome, is caused by heterozygous mutations in the STAT3 gene and is characterized by eczematoid dermatitis and recurrent skin and pulmonary infections (3). Autosomal recessive forms are mainly caused by tyrosine kinase 2 gene (TYK2) and dedicator of cytokinesis 8 (DOCK 8) mutations. DOCK 8 deficiency is often characterized by severe cutaneous viral infections and by an increased risk of malignancy at a younger age, whereas TYK2 deficient patients develop more frequently atypical mycobacterial and viral infections $(4,5)$. The definitive diagnosis is provided by genetic testing, but the lack of a general availability may lead to delayed diagnosis and treatment. There have been a number of diagnostic recommendations up to the present. Woellner, et al. established a scoring system for HIES and divided the diagnosis into possible, probable and definitive. The possible diagnosis is defined by elevated serum $\operatorname{IgE}$ above $1,000 \mathrm{IU} / \mathrm{ml}$ and a clinical score $>30$ which is based on recurrent pneumonia, newborn rash, pathologic bone fractures, characteristic face, and high palate. STAT3 mutations in addition to clinical characteristics confirm the diagnosis (6). We report a 9 year-old girl, symptomatic since 1 month and 2 weeks of age, with multisystemic involvement manifesting with recurrent infections of the skin and lungs and atypical 
facial features including broad nasal bridge and persistence of primary mandibular central incisors. The highest IgE level was $11,201 \mathrm{IU} / \mathrm{ml}$ which is highly suggestive for HIES. The diagnosis was based on hyperimmunoglobulinemia $\mathrm{E}$ and specific clinical features as genetic testing was not available in this case.

\section{CASE DESCRIPTION}

The patient, a 9 year-old girl with no history of significant disease, such as eczema or HIES in the family, was admitted to the pediatric unit with complaints of pustular lesions of the face, neck, chest, axillary, inguinal and gluteal region for a week, primarily on the face and neck and spread to the regions mentioned above. Physical examination showed multiple pustular lesions associated with itching some of which had hemorrhaged and crusted. The affected areas included: face, neck, axillary and gluteal regions. She was afebrile at the moment of presentation. Asymmetry of the face was noticed due to a swelling of the right buccal region covered by pustular lesions and crusts (fig. 1). Other facial features included antimongoloid palpebral fissures and broad nasal bridge. Oral examination revealed a high arched palate, persistence of primary mandibular central incisors and numerous dental caries (fig. 2). A postoperative scar could be observed on right hemithorax (fig. 3) due to a pulmonary cystectomy which was performed at 1 year and 7 months of age.

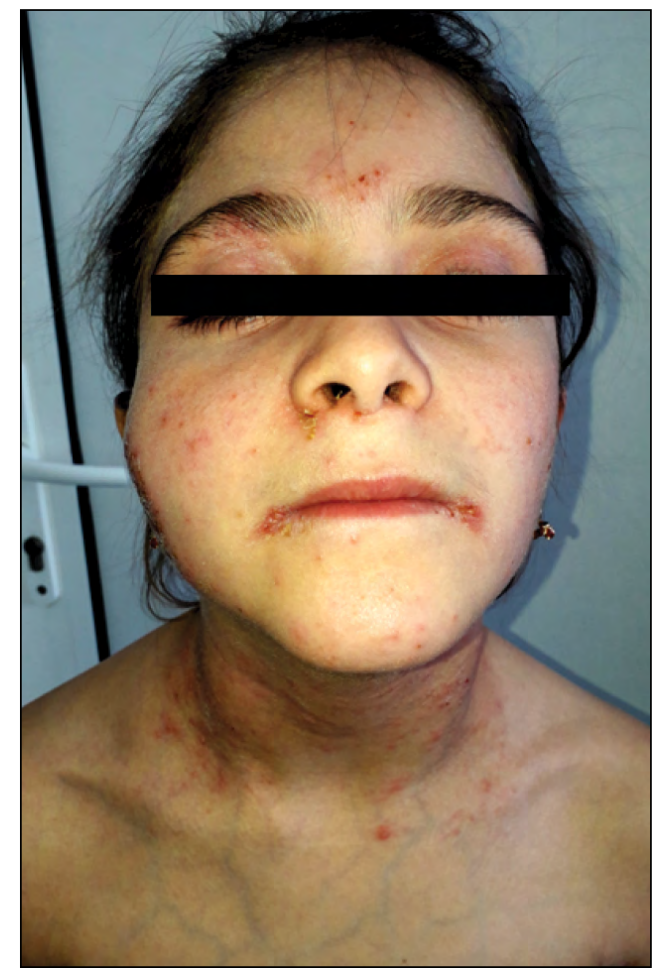

FIGURE 1. Asymmetry of the face due to a swelling of the right buccal region

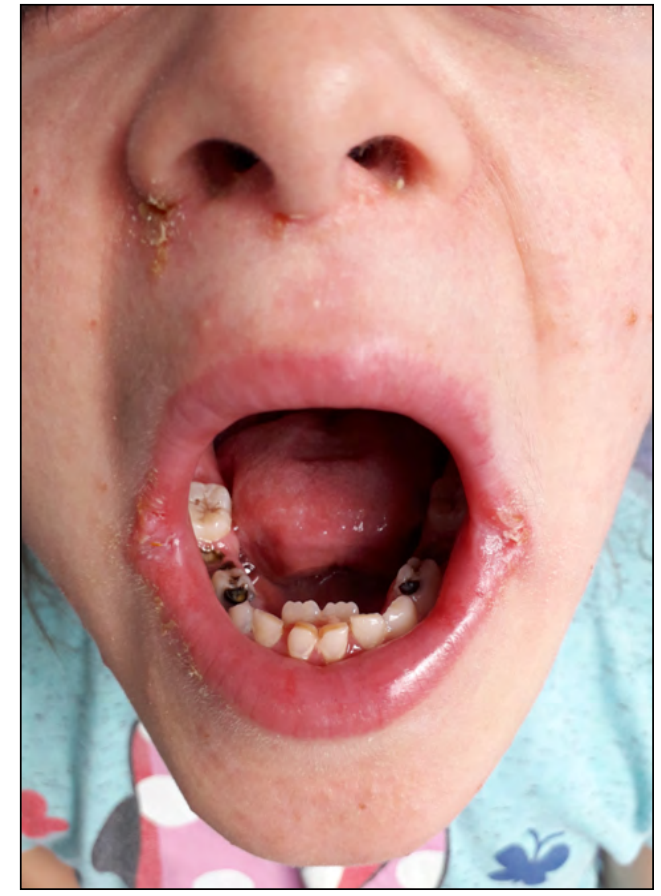

FIGURE 2. Persistence of primary mandibular central incisors

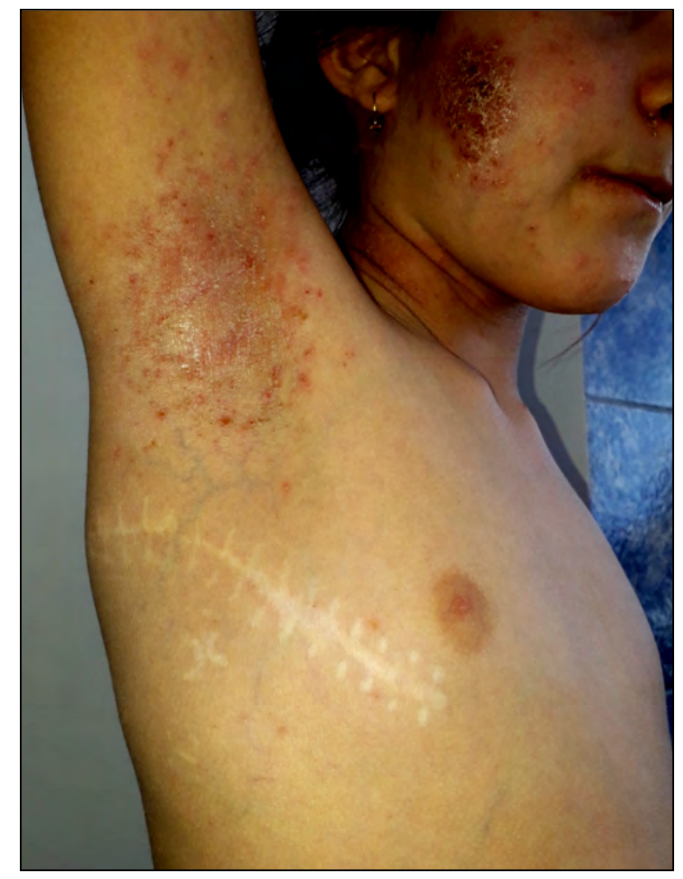

FIGURE 3. Postoperative scar on right hemithorax and pustules in the axillary region

She was born at 36 weeks gestation via normal spontaneous vaginal delivery to 40 year-old mother $8 \mathrm{G} / 5 \mathrm{P}$. According to anamnestic data, during pregnancy, the mother has been repeatedly exposed to chemicals (herbicides). She received prenatal care and standard screening tests were reportedly normal. The birth weight was 3,300 grams and the Apgar score was 7 . She required intubation and ventilation due to perinatal hypoxemia. 
She progressed well and continued to receive appropriate postnatal care until at 1 month and 2 weeks of age when she developed multiple microabscesses which were treated with Ceftriaxone according to antibiogram results (Pseudomonas aeruginosa). At 1 year and 6 months of age, the patient was admitted to hospital with dyspnea and perioronasal cyanosis. Laboratory results showed raised C-reactive protein, leucocytosis and hypergammaglobulinemia. Chest $\mathrm{X}$ ray revealed pneumothorax and a cystic lesion on the right side of the lung. One month later, she is admitted to "Grigore Alexandrescu“ Emergency Hospital for Children Bucharest with influenced general condition, pale teguments and mucosae, fever and dyspnea. Clinical examination revealed decreased vesicular breath sounds on the right hemithorax lung base and subcutaneous emphysema of the anterior and lateral right thoracic wall. Chest X-ray confirmed the same image of pulmonary cyst with chest contralateral mediastinal shift and subcutaneous emphysema. With no sign of improvement after 1 month on antibiotics, she underwent partial right cystectomy via general anesthesia. After recovering from surgery, she developed chronic eczema and multiple episodes of infections including cutaneous abscesses and chalazia which were treated according to antibiogram results (Staphyloccocus aureus).

At 3 year of age, the patient was admitted to „Sf. Maria“ Emergency Clinical Hospital for Children in Iaşi. On examination she had generalized eczematous lesions, bilateral chalazia and an abscess of the right gluteal region. Patient's facial features resembled those frequently present in HIES syndrome: prominent forehead, broad nasal bridge and deep set eyes. Her laboratory work up included excessively elevated serum level of IgE 7,587 (normal range 0-90 IU/ml), low IgA $59.5 \mathrm{mg} / \mathrm{dl}$ (normal range $83-217 \mathrm{mg} / \mathrm{dl}$ ) and eosinophilia $18.9 \%$ (normal range $0-7 \%$ ). Level of IgG and IgM were in normal range. Sweat analysis using pilocarpine iontophoresis was negative (33 $\mathrm{mmol} / \mathrm{l} \mathrm{NaCl}$ ). Mutational studies for STAT3 gene were not available but diagnostic scoring for HyperIgE syndrome according to National Institutes of Health yielded a score of 34.98 , predicting a likelihood of this mutation (Table 1). The diagnosis was based on elevated $\operatorname{IgE}$ level above 1,000 IU/ml plus a weighted score of clinical features above 30 . The primary alternative diagnosis is atopic dermatitis which is characterized by weeping, superinfected lesions, whereas recurrent abscesses are more frequently seen in HIES patients. Inflammatory causes including Kimura disease (most common in Asian men in the third decade of life with subdermal lesions or painless unilateral inflammation of cervical lymph nodes) and
Churg-Strauss syndrome (extravascular granulomatosis, small and medium vessels eosinophilic vasculitis) have been ruled out. Also, other primary immunodeficiencies have been excluded: Wiskott-Aldrich syndrome (thrombocytopenia, eczema, and recurrent infections), Netherton syndrome (eosinophilia, depressed IgG levels, ichthyosis, and atopy), Omenn syndrome (exudative erythroderma with descuamation, lymphadenopathy and hepatosplenomegaly, severe respiratory tract infections with intractable diarrea and failure to thrive), and Nezelof syndrome (atopic dermatitis, recurrent infections, pondostatural retardation, diarrhea).

TABLE 1. Diagnostic scoring for Hyper IgE syndrome in our patient

\begin{tabular}{|l|c|c|c|}
\hline \multicolumn{1}{|c|}{ Clinical features } & Points & Scale & $\begin{array}{c}\text { Scaled } \\
\text { points }\end{array}$ \\
\hline Pneumonias (>3) & 8 & 2.5 & 20 \\
\hline Newborn rash(present) & 4 & 2.08 & 8.32 \\
\hline Pathologic bone fractures (absent) & 0 & 3.33 & 0 \\
\hline $\begin{array}{l}\text { Characteristic face for Job syndrome } \\
\text { (mild) }\end{array}$ & 2 & 3.33 & 6.66 \\
\hline Cathedral palate (present) & 0 & 2.5 & 0 \\
\hline Total & \multicolumn{4}{|c|}{34.98} \\
\hline
\end{tabular}

The cutaneous lesions resolved completely without scarring using lowest strength of hydrocortisone cream. The patient was started on antibacterial prophylaxis with Trimethoprim-sulfamethoxazole and and is being followed up regularly. One year after the last admission in the hospital she developed a staphylococcal abscess of right temporal region which needed surgical drainage.

\section{DISCUSSION}

The polymorphism of manifestations in HIES leads to the difficulty of establishing the diagnosis and initiating treatment promptly. Morever, some of the most common clinical findings observed in HIES (eczema, increased serum IgE levels, recurrent skin infections), overlap with those associated with other primary immunodeficiency disorders, as well as with severe forms of atopic dermatitis. In contrast to other primary immunodeficiencies, HIES is characterized by a wide variety of nonimmunological features (7). Our patient only had the characteristic facial appearance, retained primary teeth and intraoral lesions, whereas other nonimmunological features such as hypertension, vascular abnormalities, or minimal trauma fractures were absent in this case.

The difficulties incorporating genetic testing into medical practice generated clinical scoring systems that include both immunologic/infectious manifesta- 
tions and skeletal/connective tissue abnormalities. In our case, mutational studies were not available, but clinical manifestations and positive history for recurrent infections were suggestive for diagnosis. The first clinical scoring system was developed by a research group on HIES at the USA National Institutes of Health which recognized HIES to be a multisystemic disorder (8). Nowadays diagnosis combines a high index of suspicion based on clinical features with molecular genetic testing of STAT3 $(9,10)$. Woellner et al. (6) developed guidelines that include the National Institutes of Health clinical feature scoring system as well as determination of IL-17-producing T cells. Elevated total IgE concentrations greater than 1,000 IU/ $\mathrm{ml}$ and a weighted score of greater than 30 indicate a defect in STAT3 gene. In our patient, a score of 34.98 points was highly suggestive for STAT3 mutation. In most cases, HIES patients have normal or decreased serum IgM, IgG and IgA levels (11). In our patient, IgG and IgM levels were within normal range, but IgA level was lower than normal values, which might suggest the immaturity of immune system.

At present, there is no curative treatment for the underlying defect in HIES. The consensus is to give life-long prophylactic therapy with anti-staphylococcal antibiotic such as trimethoprim-sulfamethoxazole

\section{REFERENCES}

1. Davis SD, Schaller J, Wedgwood RJ. Job's Syndrome. Recurrent, "cold", staphylococcal abscesses. Lancet. 1966;1(7445):1013-5.

2. Buckley RH, Wray BB, Belmaker EZ et al. Extreme hyperimmunoglobulinemia $E$ and undue susceptibility to infection. Pediatrics. 1972;49(1):59-70.

3. Holland SM, DeLeo FR, Elloumi HZ et al. STAT3 mutations in the hyper-IgE syndrome. N Engl J Med. 2007;357(16):1608-1619.

4. Minegishi Y, Saito M, Morio T et al. Human Tyrosine Kinase 2 Deficiency Reveals Its Requisite Roles in Multiple Cytokine Signals Involved in Innate and Acquired Immunity. Immunity. 2006;25:745755.

5. Engelhardt KR, McGhee S, Winkler $S$ et al. Large deletions and point mutations involving the dedicator of cytokinesis 8 (DOCK8) in the autosomal-recessive form of hyper-lgE syndrome. J Allergy Clin Immunol. 2009;124:1289-1302.

6. Woellner C, Gertz EM, Schäffer AA et al. Mutations in STAT3 and diagnostic guidelines for hyper-IgE syndrome. J Allergy Clin Immunol. 2010;125:424-432.

7. Freeman AF, Holland SM. Clinical manifestations, etiology, and pathogenesis of the hyper-lgE syndromes. Pediatr Res. 2009; 65:R32-7.

8. Grimbacher B, Schäffer AA, Holland SM et al. Genetic linkage of hyper-IgE syndrome to chromosome 4. Am J Hum Genet. 1999; 65:735-44.

9. Holland SM, DeLeo FR, Elloumi HZ et al. STAT3 mutations in the hyper-IgE syndrome. N Engl J Med. 2007;357:1608-19.

10. Minegishi Y, Saito M, Tsuchiya S et al. Dominant-negative mutations in the DNA-binding domain of STAT3 cause hyper-IgE syndrome. Nature. 2007; 448:1058-62.
(12). IVIG may be able to decrease the number of infections (13). The role of bone marrow transplantation still remains controversial as the first reported case was followed by reappearance of manifestations and of increased serum IgE levels (14). Children need close follow up for appropriate management of infections and surveillance for malignancies and autoimmune diseases. HIES is associated with an increased risk of systemic lupus erythemathosus (15), dermatomyositis (16), and membranoproliferative glomerulonephritis (17). An increased incidence of lymphoproliferative disorders has also been reported particularly of non-Hodgkin lymphomas (18) and of Hodgkin disease (19).

\section{CONCLUSIONS}

This case emphasizes that paediatricians should consider the diagnosis of primary immunodeficiency in children who are symptomatic since early infancy and present recurrent infections. Diagnosis of HIES is still challenging as we face many difficulties in accessing molecular genetic tests. In our case, the recurrence of cutaneous abscesses despite prophylactic antibiotherapy highlights the importance of constant vigilance regarding infectious complications.

11. Buckley RH. The hyper-lgE syndrome. Clin Rev Allergy Immunol. 2001;20(1):139-154.

12. 12.Grimbacher B, Holland SM, Puck JM. Hyper-lgE syndromes. Immunol Rev. 2005;203:244-0.

13. Wakim M, Alazard M, Yajima A et al. High dose intravenous immunoglobulin in atopic dermatitis and hyper-IgE syndrome. Ann Allergy Asthma Immunol. 1998;81:153-8.

14. Gennery A, Flood T, Abinun M et al. Bone marrow transplantation does not correct the hyper IgE syndrome. Bone Marrow Transplant. 2000;25:1303-1305.

15. Perazzio SF, Granados A, Salomao R et al. High frequency of immunodeficiency-like states in systemic lupus erythematosus: A cross-sectional study in 300 consecutive patients. Rheumatol. 2016;55:1647-1655.

16. Patel NH, Padhiyar JK, Shah YB et al. Unusual presentations and associations of hyper IgE Syndrome: Retrospective analysis of ten cases at tertiary care institute - With review of indian published reports. Indian J Paediatr Dermatol. 2018;19:31-6.

17. Tanji C, Yorioka N, Kanahara K et al. Hyperimmunoglobulin $\mathrm{E}$ syndrome associated with nephrotic syndrome. Intern Med. 1999;38:491-494.

18. Onal IK, Kurt M, Altundag K et al. Peripheral T-cell lymphoma and Job's syndrome: a rare association. Med Oncol. 2006;23:141-144.

19. Kashef MA, Kashef S, Handjani F et al. Hodgkin lymphoma developing in a 4.5-year-old girl with hyper-IgE syndrome. Pediatr Hematol Oncol. 2006;23:59-63.

Conflict of interest: none declared Financial support: none declared 